\title{
Extração de biomassa aderida ao meio-suporte de um reator de leito móvel com biofilme: agitação mecânica e ultrassom
}

\author{
Extraction of biomass attached to a support media in a \\ moving bed biofilm reactor: mechanical agitation and ultrasound
}

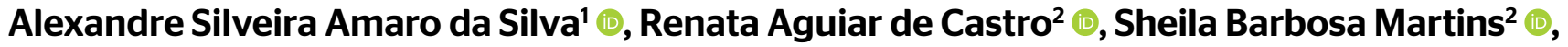
Rodrigo Derossi Alvim ${ }^{2} \odot$, André Luís de Sá Salomão ${ }^{\oplus}$, Marcia Marques ${ }^{1 *}($ ()
\end{abstract}

$\square$

\section{RESUMO}

A quantificação de biomassa fixa em reatores de leito móvel com biofilme (MBBRs) é essencial para avaliação e controle do desempenho deles. Entretanto, observa-se que tal quantificação é realizada empregando-se diversas técnicas, o que dificulta comparações entre resultados. No presente trabalho, foram testadas duas das principais técnicas (agitação mecânica e ultrassom) utilizadas para extração de biomassa aderida aos meios-suporte (mídias) de um MBBR sem utilização de agentes químicos. Os desenhos experimentais foram realizados utilizando-se planejamento fatorial completo, e, para tal, foram selecionadas duas variáveis independentes: tempo (T) de exposição de 15 a 60 min; e volume de água destilada utilizado para extração (V) de 100 a 400 mL. O ultrassom mostrou-se superior à agitação mecânica quanto à eficiência no percentual extraído de sólidos suspensos totais (\%SST), e embora nenhuma das variáveis ( $\mathrm{T}$ ou V) tenha contribuído significativamente nas faixas testadas, com base na superfície de respostas, foi conduzido um novo experimento, ampliando a faixa de T de 5 a 90 min. A variável T contribuiu significativamente para o \%SST, e o melhor resultado foi obtido com $\mathrm{T}=90 \mathrm{~min}$. Recomenda-se o uso de ultrassom, volume de mais fácil manuseio $(250 \mathrm{~mL})$ e $\mathrm{T}=90 \mathrm{~min}$. Ensaios adicionais são recomendados, tendo em vista a padronização do método. Palavras-chave: esgoto; MBBR; meios-suporte; biomassa fixa; sólidos suspensos totais; ultrassom.

\begin{abstract}
Quantification of attached biomass in Moving Bed Biofilm Reactors (MBBR) is essential for performance assessment and process control. However, it is observed that such quantification is performed using the most diverse techniques, which hampers comparisons between results. In this investigation, two of the main techniques used (mechanical agitation and ultrasound) were compared for removal of the biomass attached to the support media of an MBBR reactor without the use of chemical agents. The experiments were planned following full factorial designs using the two independent variables: Exposure time ( $\mathrm{T}$ ) from 15 to $60 \mathrm{~min}$; and Volume of distillate water used for extraction (V) from 100 to $400 \mathrm{~mL}$. The results revealed that ultrasound is superior to mechanical agitation regarding detachment of the total suspended solids (\%TSS) and though neither $\mathrm{T}$ or $\mathrm{V}$ contributed significantly within the interval tested, based on the response surface, a new experiment was performed using an increased $\mathrm{T}$ range from 5 to $90 \mathrm{~min}$. Then, exposure time contributed significantly to the \%TSS detachment, with the best result at 90 min. The use of ultrasound is therefore recommended, with volume that is easier to handle (250 mL) and time equal to $90 \mathrm{~min}$. Additional tests are recommended for method standardization.
\end{abstract}

Keywords: sewage; MBBR; support media; fixed biomass; total suspended solids; ultrasound.

\section{INTRODUÇÃO}

A tecnologia do reator de leito móvel com biofilme (moving bed biofilm reactor - MBBR) foi desenvolvida na década de 1980 na Noruega, com o objetivo de aumentar a eficiência do tratamento de efluentes em plantas convencionais sem que fosse necessário ampliá-las, aumentando-se a densidade populacional microbiana em reatores convencionais por meio da utilização de meios-suporte (mídias), sobre os quais se desenvolve o biofilme (RUSTEN et al., 2006; ANDREOTTOLA et al., 2000; ØDEGAARD, 2006). Dessa forma, os MBBRs podem ser classificados como sistemas do tipo híbrido, uma vez que a biomassa formada

'Departamento de Engenharia Sanitária e do Meio Ambiente, Universidade do Estado do Rio de Janeiro - Rio de Janeiro (RJ), Brasil.

${ }^{2}$ Companhia Estadual de Águas e Esgotos - Rio de Janeiro (RJ), Brasil.

*Autor correspondente: marciamarques@eng.uerj.br

Recebido: 30/08/2018 - Aceito: 10/09/2019 - Reg. Abes: 20180113 
por microrganismos está presente tanto na forma suspensa quanto na forma fixa — biofilme (JORDÃO; PESSÔA, 2014) —, podendo ser utilizados tanto para processos aeróbios quanto para processos anóxicos ou anaeróbios (BARWAL; CHAUDHARY, 2014).

Além de fornecer maior área superficial para desenvolvimento de microrganismos, é necessário também que as mídias estejam em constante movimento, o que favorece o contato entre os compostos presentes no meio líquido e o biofilme (ØDEGAARD, 2006). Nos reatores aerados o movimento é promovido pelas bolhas de ar (Figura 1A), e nos reatores anóxicos e anaeróbios, por agitadores mecânicos (Figura 1B).

Uma das principais variáveis para se avaliar o desempenho desse tipo de sistema é o teor de biomassa aderida às mídias. Portanto, sua quantificação é fundamental para o controle do processo. Tal quantificação é realizada por meio da extração do biofilme das mídias que, em seguida, é submetido às análises da série de sólidos por métodos gravimétricos, conforme American Public Health Association, American Water Works Association e Water Environment Federation (APHA, AWWA e WEF, 2017). Várias formas de extração estão disponíveis na literatura, tais como agitação (FERRAI; GUGLIELMI; ANDREOTTOLA, 2010; DI BIASE et al., 2018), ultrassom (NOGUEIRA, 2013; WANG; WEN; QIAN, 2005; OLIVEIRA, 2008), raspagem manual (MAHENDRAN; LISHMAN; LISS, 2012; ZHANG et al., 2013a; ZHANG et al., 2013b; WANG et al., 2018), lavagem (OLIVEIRA, 2008) e agentes químicos (OLIVEIRA, 2008; LEVSTEK; PLAZL, 2009; SHORE et al., 2012; FALÅS et al., 2013; CAO, 2014; BASSIN et al., 2016; ABTAHI et al., 2018; OOI et al., 2018). Porém, não existe ainda na literatura uma metodologia padrão recomendada para a extração da biomassa das mídias, bem como não existe padronização sobre o tamanho do poro das membranas usadas nas análises de sólidos suspensos, o que pode levar a diferentes conclusões sobre o teor de biomassa nesse tipo de reator.

O presente trabalho teve por objetivos:

- Comparar duas técnicas de extração dos sólidos aderidos às mídias (agitação mecânica versus ultrassom);

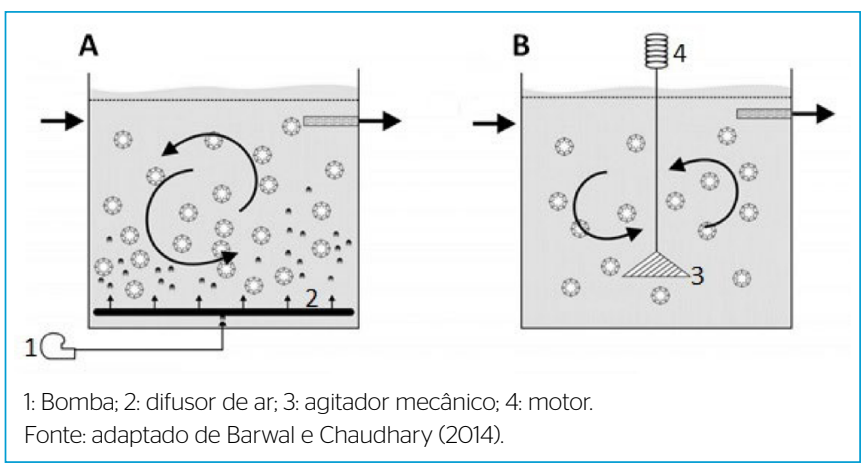

Figura 1 - Funcionamento dos reatores de leito móvel com biofilme. (A) Reator aeróbio; (B) reator anaeróbio.
- Verificar as diferenças nos resultados da quantificação de sólidos aderidos em função das membranas utilizadas com poros de $0,7 \mathrm{e}$ $1,5 \mu \mathrm{m}$.

\section{MATERIAIS E MÉTODOS}

As mídias foram coletadas no tanque aerado de um reator do tipo MBBR operado pelo grupo de pesquisa, o qual está instalado em uma elevatória de esgoto municipal da empresa estadual de água e esgotos. Esse MBBR corresponde à etapa aerada de um sistema do tipo BARDENPHO, que é composto de cinco unidades ou etapas (anaeróbia, anóxica, aerada, anóxica e sedimentação).

O sistema é alimentado com esgoto bruto, em regime contínuo, e a mistura completa nos reatores é promovida ora por agitadores mecânicos (etapa anaeróbia e anóxica), ora pela agitação das bolhas de ar (etapa aeróbia). Completam a configuração BARDENPHO as recirculações de efluente e de lodo entre os tanques aerado e anóxico-1 (pré-desnitrificação) e; o sedimentador anaeróbio.

O volume do reator aerado é de $240 \mathrm{~L}$, sendo $50 \%$ do seu interior preenchido com mídias que ocupam volume de aproximadamente 18 L. Portanto, o volume útil do reator é igual a $222 \mathrm{~L}$.

\section{Características das mídias}

As mídias adotadas neste estudo foram peças plásticas com formato cilíndrico da marca Enviromex, fabricado em PEAD (polietileno de alta densidade), de cor branca, com diâmetro externo de $26 \mathrm{~mm}$, densidade de $0,96 \mathrm{~g} / \mathrm{cm}^{3}$ e área específica de $500 \mathrm{~m}^{2} / \mathrm{m}^{3}$.

\section{Planejamento experimental}

Este estudo foi dividido em duas etapas:

- Etapa I: comparação entre agitação mecânica e ultrassom;

- Etapa II: ampliação do tempo de exposição ao ultrassom e comparação entre membranas com porosidade 0,7 e 1,5 $\mu \mathrm{m}$.

Em ambas as etapas foi empregado planejamento experimental fatorial (Design of Experiment - DoE) para otimização de processos (RODRIGUES; IEMMA, 2009), de modo que os desenhos experimentais foram compostos de diferentes combinações entre as principais variáveis independentes que influenciam cada técnica, sendo elas:

- Tempo (T);

- Volume de água destilada utilizado para extração (V).

Para cada variável o intervalo investigado foi dividido em 3 níveis, resultando em 5 diferentes combinações mais triplicata (Etapa I) ou quintuplicata (Etapa II) no ponto central, resultando em 7 ensaios para cada técnica (14 ensaios na Etapa I - Tabela 1) e em 9 ensaios para cada tipo de membrana (totalizando 18 ensaios na Etapa II - Tabela 2). 
Tabela 1 - Etapa I (Blocos 1 e 2): comparação entre ultrassom e agitação mecânica para extração de biofilme de mídias do reator de leito móvel com biofilme.

\begin{tabular}{|c|c|c|c|c|}
\hline Bloco 1 & Bloco 2 & \multirow{2}{*}{$\begin{array}{l}\text { Niveis das } \\
\text { variáveis } \\
\text { combinadas }\end{array}$} & \multicolumn{2}{|c|}{ Variáveis } \\
\hline $\begin{array}{l}\text { Ultrassom } \\
224^{\circ} \mathrm{C}\end{array}$ & $\begin{array}{l}\text { Agitação } \\
\text { mecânica } \\
24^{\circ} \mathrm{C}\end{array}$ & & $\begin{array}{l}\text { Tempo } \\
\text { (min) }\end{array}$ & $\begin{array}{l}\text { Volume } \\
(\mathrm{mL})\end{array}$ \\
\hline A & $\mathrm{H}$ & $(-1,-1)$ & 15 & 100 \\
\hline B & I & $(+1,-1)$ & 60 & 100 \\
\hline C & J & $(-1,+1)$ & 15 & 400 \\
\hline D & K & $(+1,+1)$ & 60 & 400 \\
\hline E & L & $(0,0)$ & 37,5 & 250 \\
\hline $\mathrm{F}$ & M & $(\mathrm{O}, \mathrm{O})$ & 37,5 & 250 \\
\hline G & N & $(\mathrm{O}, \mathrm{O})$ & 37,5 & 250 \\
\hline
\end{tabular}

Tabela 2 - Etapa II: comparação entre duas membranas de diferentes porosidades (Bloco 1: 0,7 $\mu \mathrm{m}$; Bloco 2:1,5 $\mu \mathrm{m}$ ) na extração e quantificação da biomassa (\%SST) de mídias do reator de leito móvel com biofilme, utilizando ultrassom com tempo de 5 a 90 min.

\begin{tabular}{|c|c|c|c|c|}
\hline Bloco 1 & Bloco 2 & & & \\
\hline $\begin{array}{c}\text { Ensaios com } \\
\text { membrana } \\
0,7 \mu \mathrm{m}\end{array}$ & $\begin{array}{c}\text { Ensaios com } \\
\text { membrana } \\
1,5 \mu \mathrm{m}\end{array}$ & $\begin{array}{l}\text { Niveis combinados } \\
\text { das variáveis }\end{array}$ & $\begin{array}{c}\text { Tempo } \\
\text { (min) }\end{array}$ & $\begin{array}{l}\text { Volume } \\
(\mathrm{mL})\end{array}$ \\
\hline A & $\mathrm{J}$ & $(-1,-1)$ & 5 & 100 \\
\hline B & K & $(+1,-1)$ & 90 & 100 \\
\hline C & L & $(-1,+1)$ & 5 & 400 \\
\hline D & M & $(+1,+1)$ & 90 & 400 \\
\hline$E$ & $N$ & $(0,0)$ & 47,5 & 250 \\
\hline $\mathrm{F}$ & O & $(0,0)$ & 47,5 & 250 \\
\hline G & $P$ & $(\mathrm{O}, \mathrm{O})$ & 47,5 & 250 \\
\hline $\mathrm{H}$ & Q & $(0,0)$ & 47,5 & 250 \\
\hline I & $\mathrm{R}$ & $(\mathrm{O}, \mathrm{O})$ & 47,5 & 250 \\
\hline
\end{tabular}

\section{Amostragem das unidades de mídias}

Em cada um dos 32 frascos utilizados neste estudo foram adicionadas 6 unidades de mídias, que após coleta aleatória foram imediatamente acondicionadas em caixa térmica $\left(0 \mathrm{a} 4^{\circ} \mathrm{C}\right)$ até o momento dos ensaios. Durante a coleta das mídias, tomou-se o cuidado de selecionar aquelas que aparentassem ter diâmetro e forma mais parecidos possível, já que existe diferença visível nesses aspectos em decorrência de variações nos processos de fabricação e armazenamento.

\section{Etapa I: agitação mecânica e ultrassom}

Para ambas as técnicas foram usados os mesmos intervalos de variáveis independentes, sendo o $\mathrm{T}$ de tratamento (agitação mecânica e ultrassom) de 15 a 60 min; e o V de água destilada nos frascos entre 100 e $400 \mathrm{~mL}$ com desenho experimental, conforme a Tabela 1. Para a agitação mecânica, os frascos foram submetidos à agitação contínua em mesa agitadora a $200 \mathrm{rpm}$ em movimento orbital, enquanto para o ultrassom $(40 \mathrm{kHz})$ os frascos foram removidos a cada 5 min e agitados manualmente por $3 \mathrm{~s}$ ao longo do processo de sonicação de cada ensaio.

\section{Etapa II: ultrassom, expansão do tempo, membranas distintas $(0,7$ e $1,5 \mu \mathrm{m})$}

O desenho experimental desta etapa foi elaborado em função dos resultados obtidos na Etapa I, de modo que a técnica empregada para extração de biofilme das mídias foi o ultrassom, e o intervalo investigado para a variável $\mathrm{T}$ foi ampliado de 15-60 para 5-90 min, conforme a Tabela 2. Além disso, foram adicionados mais dois ensaios ao ponto central (quintuplicata). Quanto à agitação manual, nesta etapa os frascos foram agitados apenas 3 vezes, por $3 \mathrm{~s}$ cada vez e em intervalos de tempo iguais, ao longo do processo de ultrassom.

\section{Quantificação da biomassa extraída}

Para a realização dos ensaios foram utilizados frascos de $500 \mathrm{~mL}$ preenchidos com os volumes de água destilada descritos anteriormente, e após extração (via agitação mecânica ou ultrassom), as mídias foram retiradas dos frascos e colocadas em frasco separado para análise posterior. A fração líquida de cada amostra foi analisada com relação aos sólidos suspensos totais (SST), segundo método 2540D (APHA; AWWA; WEF, 2017), e com as mídias foi realizado o procedimento de extração química com hidróxido de sódio (1 M) para remoção do biofilme remanescente, conforme rotina própria desenvolvida para o presente estudo.

\section{Análise da fração líquida}

Após aplicação de ultrassom ou agitação, cada amostra foi colocada em um Becker e homogeneizada, com o auxílio de um misturador, por $60 \mathrm{~s}$. Em seguida, uma alíquota $(10 \mathrm{~mL})$ de cada frasco foi filtrada. Os filtros utilizados neste trabalho foram das marcas Sartorius (modelo MG 550-HA, de 0,30 $\mathrm{mm}$ de espessura, $47 \mathrm{~mm}$ de diâmetro e porosidade 1,5 $\mu \mathrm{m}$ ) e Merck (modelo AP4004705 com 0,475 mm de espessura, $47 \mathrm{~mm}$ de diâmetro e porosidade $0,7 \mu \mathrm{m}$ ), ambos fabricados em fibra de vidro.

Após as filtrações, as membranas foram colocadas em estufa por $1 \mathrm{~h}\left(103-105^{\circ} \mathrm{C}\right)$ e, então, dispostas no dessecador por $1 \mathrm{~h}$ para seu resfriamento. Em seguida, foram pesadas individualmente e colocadas novamente na estufa por $1 \mathrm{~h}$, repetindo o processo. Após aferição do peso constante em estufa, o filtro com o resíduo seco foi levado à mufla por $15 \mathrm{~min}$ para calcinação a $550 \pm 50^{\circ} \mathrm{C}$, sendo, em seguida, retirado da mufla e colocado no dessecador por $1 \mathrm{~h}$ para seu resfriamento e pesagem. Esse processo foi repetido até aferição de peso constante.

Os sólidos remanescentes representam os sólidos em suspensão fixos (SSF), enquanto a massa perdida na calcinação representa os sólidos em suspensão voláteis (SSV). Para este trabalho, foram considerados sólidos totais aderidos às mídias $\left(\mathrm{STA}_{\mathrm{m}}\right)$ o resultado da análise de 
SST convertido para o volume de frasco de onde foi extraída alíquota mais o somatório da massa que ficou aderida à mídia após o processo de extração (Equação 1).

$S T A_{m}=M A_{m}+S S T$

Em que:

$\mathrm{STA}_{\mathrm{m}}=$ sólidos totais aderidos às mídias;

$\mathrm{MA}_{\mathrm{m}}=$ massa de sólidos aderida às mídias;

SST = sólidos suspensos totais.

O cálculo da eficiência de extração $\left(\mathrm{E}_{\text {ext }}\right)$ foi realizado por meio da divisão da massa de sólidos extraídos pela massa dos $\mathrm{STA}_{\mathrm{m}}$ (Equação 2).

$E_{\text {ext }}=100 \frac{S S T}{S S T+S T A_{m}}$

Em que:

$\mathrm{E}_{\text {ext }}=$ eficiência de extração;

SST = sólidos suspensos totais;

$\mathrm{STA}_{\mathrm{m}}=$ sólidos totais aderidos às mídias.

\section{Cálculo da massa de sólidos aderida às mídias}

As mídias separadas após os processos de extração (ultrassom ou agitação mecânica) foram colocadas em pratos de alumínio e levadas à estufa por $24 \mathrm{~h}\left(103-105^{\circ} \mathrm{C}\right)$. Em seguida, foram colocadas no dessecador para resfriamento por $60 \mathrm{~min}$ antes da pesagem. Esse peso foi considerado como massa das mídias após extração $\left(\mathrm{MM}_{\text {ext }}\right)$, que corresponde ao somatório da massa de biofilme remanescente após processo de extração com a massa das próprias mídias.

Após pesagem, cada grupo de mídias foi colocado em frascos Erlenmeyer de $250 \mathrm{~mL}$ contendo $100 \mathrm{~mL}$ da solução hidróxido de sódio (1 M). Tais frascos foram posicionados em placa aquecedora (Quimis modelo Q313A) por $30 \mathrm{~min}$, sendo os primeiros $15 \mathrm{~min}$ a $300^{\circ} \mathrm{C}$ e os últimos 15 min a $150^{\circ} \mathrm{C}$. Durante o aquecimento, os frascos Erlenmeyer foram agitados em três ocasiões: aos 10, 20 e $30 \mathrm{~min}$.

As mídias foram lavadas em duas etapas. Na primeira etapa, água de torneira foi adicionada aos frascos Erlenmeyer até o cobrimento das mídias e, em seguida, os frascos foram agitados manualmente por aproximadamente $5 \mathrm{~s}$. Essa etapa foi repetida dez vezes para cada grupo de peças. Na segunda etapa, água destilada foi adicionada até o cobrimento das mídias e, em seguida, os frascos foram agitados manualmente por aproximadamente $5 \mathrm{~s}$. Essa etapa foi repetida três vezes para cada Erlenmeyer. Após lavagem, as mídias foram posicionadas em pratos de alumínio e levadas à estufa por mais $24 \mathrm{~h}$.

Após $24 \mathrm{~h}$ em secagem, cada grupo de mídias foi pesado para aferição do peso das mídias sem o biofilme remanescente. Tal peso foi denominado "massa das mídias após lavagem" $\left(\mathrm{MM}_{\mathrm{lav}}\right)$. A massa de sólidos aderida às mídias $\left(\mathrm{MA}_{\mathrm{m}}\right)$ foi calculada pela diferença do peso inicial por esse peso após lavagem (Equação 3).

$M A_{m}=M M_{\text {ext }}-M M_{\text {lav }}$

Em que:

$\mathrm{MA}_{\mathrm{m}}=$ massa de sólidos aderida às mídias;

$\mathrm{MM}_{\text {ext }}=$ massa das mídias após extração;

$\mathrm{MM}_{\text {lav }}=$ massa das mídias após lavagem.

\section{Análises estatísticas}

Os gráficos referentes aos resultados do planejamento experimental (diagrama de Pareto, superfície de respostas etc.) foram gerados pela versão trial do software Statistica (versão trial). A eficiência de extração de biomassa das mídias por cada técnica testada é expressa em percentual extraído de sólidos suspensos totais (\%SST). As eficiências foram comparadas pelo teste de Mann-Whitney utilizando-se o software de estatística Minitab18 ${ }^{\circledR}$. Esse teste não paramétrico compara as medianas de duas amostras independentes selecionadas de populações que apresentem uma mesma distribuição.

\section{RESULTADOS}

\section{Etapa I - Extração de biomassa: ultrassom e agitação mecânica}

A eficiência de extração de biomassa das mídias, apresentada como \%SST, tanto com aplicação de ultrassom (Bloco 1) quanto com agitação mecânica (Bloco 2), e o uso de membrana de 1,5 $\mu \mathrm{m}$ resultaram em eficiências de extração, que variaram de 33 a 43\% pela agitação mecânica e de 56 a 70\% pelo ultrassom (Tabela 3) - resultados semelhantes aos

Tabela 3 - Etapa I: comparação de técnicas de extração de biomassa aderida às mídias do reator de leito móvel com biofilme. Bloco 1: ultrassom; Bloco 2: agitação mecânica. Planejamento com duas variáveis independentes $(\mathrm{k}=2)$, três níveis cada e triplicata no ponto central (Ensaios 5, 6 e 7).

\begin{tabular}{l|c|c|c|c} 
Ensaio & $\begin{array}{c}\text { Volume } \\
(\mathrm{mL})\end{array}$ & $\begin{array}{c}\text { Tempo } \\
(\mathrm{min})\end{array}$ & $\begin{array}{c}\text { Bloco 1 } \\
\text { (\%SST por } \\
\text { ultrassom) }\end{array}$ & $\begin{array}{c}\text { Bloco 2 } \\
\text { (\%SST por } \\
\text { agitação } \\
\text { mecânica) }\end{array}$ \\
\hline 1 & $100(-1)$ & $15(-1)$ & 56 & 38 \\
\hline 2 & $400(+1)$ & $15(-1)$ & 59 & 43 \\
\hline 3 & $100(-1)$ & $60(+1)$ & 66 & 33 \\
\hline 5 & $400(+1)$ & $60(+1)$ & 65 & 41 \\
\hline 6 & $250(0)$ & $37,5(0)$ & 73 & 41 \\
\hline 7 & $250(0)$ & $37,5(0)$ & $64^{*}$ & 41 \\
\hline
\end{tabular}

\%SST: percentual extraído de sólidos suspensos totais; *Outlier. 
obtidos por Oliveira (2008), que observou 64\% de extração ao aplicar sonicação de 1 h e $250 \mathrm{~mL}$ de água.

$\mathrm{Na}$ extração por ultrassom (Etapa I, Bloco 1) não houve efeito significativo $(\alpha=0,05)$ das variáveis $\mathrm{V}$ e T nos níveis estudados (Figuras 2 e 3). Na análise de variância, a falta de ajuste ao modelo não foi significativa ( $\mathrm{p}=0,1654)$ e o modelo, portanto, foi considerado válido.

Para agitação mecânica (Etapa I, Bloco 2), apenas a variável volume foi considerada significativa $(\alpha=0,05)$ (Figura 4 e 5 ). Para um mesmo tempo, o aumento do volume influenciou positivamente a eficiência de \%SST (Figura 5). A análise da variância indicou que o modelo apresenta ajuste satisfatório.

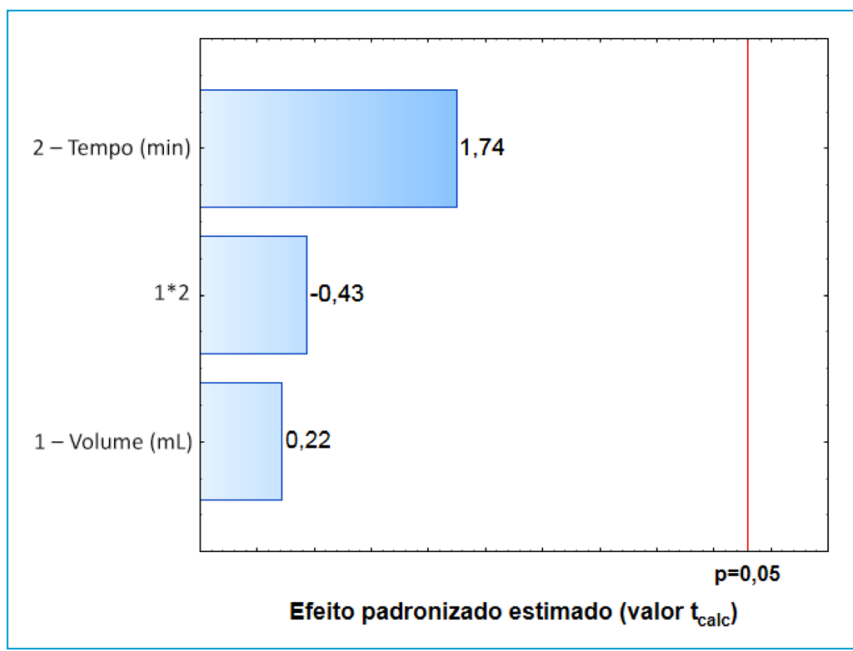

Figura 2 - Diagrama de Pareto. Estimativa do efeito padronizado das variáveis volume $(\mathrm{mL})$, tempo ( $\mathrm{min})$ e interação volume-tempo (Etapa I, Bloco 1 - Ultrassom).
No que diz respeito ao \%SST por ultrassom e por agitação mecânica, quando as medianas foram comparadas pelo teste de Mann-Whitney, utilizando-se o Minitab1 ${ }^{\circledR}$, concluiu-se que o ultrassom teve desempenho significativamente superior à agitação mecânica $(\mathrm{p}<0,05)$.

Por esse motivo, decidiu-se pela realização de um novo experimento somente com ultrassom, estendendo a faixa de tempos de exposição e utilizando, além da membrana de $1,5 \mu \mathrm{m}$, uma membrana com porosidade $0,7 \mu \mathrm{m}$ para verificar eventual influência da malha sobre o \%SST.

\section{Etapa II: extração por ultrassom em uma faixa mais ampla de tempo e com uso de dois filtros com porosidades distintas $(0,7$ e 1,5 $\mu \mathrm{m})$}

Tendo em vista a dispersão de dados no ponto central observada na Etapa I, na Etapa II, ao invés de triplicatas, foram realizadas quintuplicatas nesse ponto (Tabela 4).

A eficiência de extração do biofilme com ultrassom, cuja fração líquida foi filtrada em membrana $0,7 \mu \mathrm{m}$, ficou compreendida na faixa de 55 a 75\%, e para fração líquida que foi filtrada em membrana de $1,5 \mu \mathrm{m}$, essa faixa variou de 55 a 76\% (Tabela 4), não havendo diferença significativa entre os resultados $(\alpha=0,05)$ - como, aliás, a grande similaridade dos dados sugerem. Portanto, os dados a seguir se referem apenas à extração com membrana $1,5 \mu \mathrm{m}$, pela maior facilidade de filtração.

Neste estudo, foi possível observar que o T se destacou como variável significativa $(p=0,02)$. Com relação ao $V$, não houve contribuição significativa sobre a eficiência de extração do biofilme $(\mathrm{p}=0,09)$. Porém, ao comparar a eficiência de extração durante o mesmo tempo (por exemplo, $5 \mathrm{~min}$ ) para volumes distintos (por exemplo, 100 e $400 \mathrm{~mL}$ ), observa-se que, numericamente, a extração em volume menor foi ligeiramente

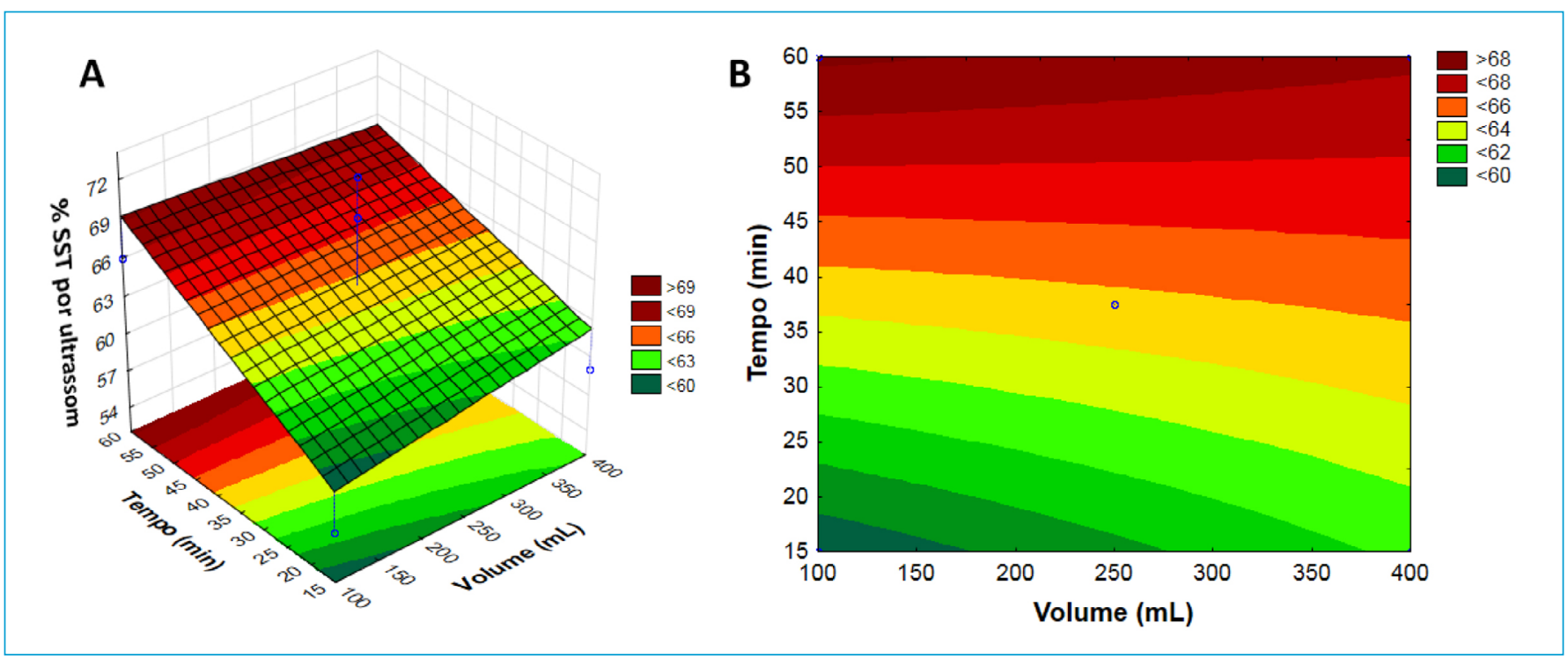

Figura 3 - Superfícies de resposta. Variáveis volume $(\mathrm{mL})$, tempo $(\mathrm{min})$ e percentual extraído de sólidos suspensos totais (\%SST) (Etapa I, Bloco 1 Ultrassom). 


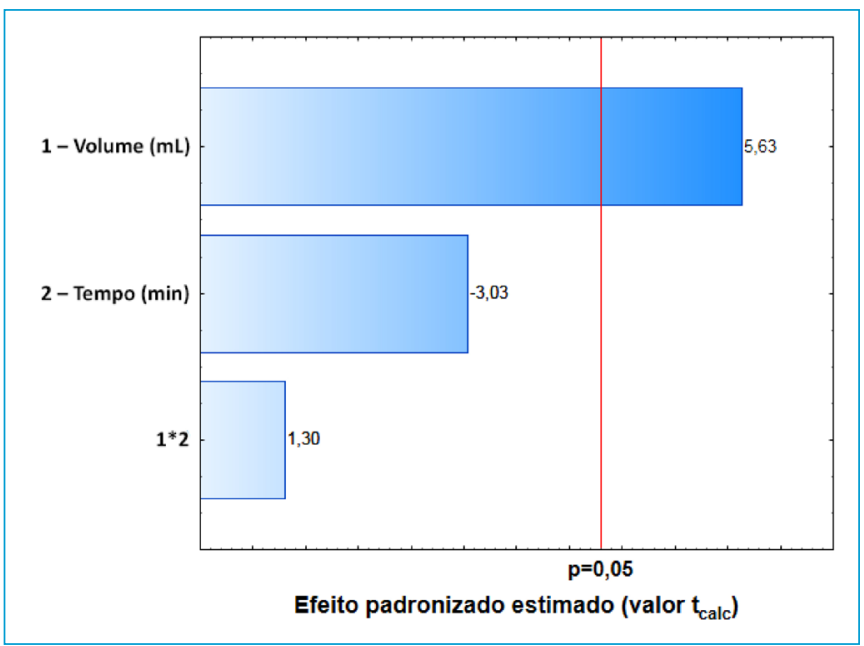

Figura 4 - Diagrama de Pareto. Efeito padronizado das variáveis volume $(\mathrm{mL})$, tempo $(\mathrm{min})$ e interação volume-tempo (Etapa I, Bloco 2 - Agitação mecânica).
Tabela 4 - Etapa II: extração de sólidos suspensos totais das mídias do reator de leito móvel com biofilme. Planejamento fatorial com duas variáveis independentes $(\mathrm{k}=2)$ e três níveis para cada variável. Quintuplicata no ponto central (Ensaios 5 a 9) e uso de duas membranas distintas (de 0,7 e $1,5 \mu \mathrm{m}$ ).

\begin{tabular}{l|c|c|c|c}
\hline Ensaios & Volume (mL) & Tempo (min) & \%SST (0,7 $\mu \mathrm{m})$ & \%SST $(1,5 \mu \mathrm{m})$ \\
\hline 1 & $100(-1)$ & $5(-1)$ & 62 & 62 \\
\hline 2 & $100(-1)$ & $90(+1)$ & 75 & 76 \\
\hline 3 & $400(+1)$ & $5(-1)$ & 55 & 55 \\
\hline 5 & $400(+1)$ & $90(+1)$ & 70 & 69 \\
\hline 6 & $250(0)$ & $47,5(0)$ & 69 & 67 \\
\hline 7 & $250(0)$ & $47,5(0)$ & 66 & 67 \\
\hline 8 & $250(0)$ & $47,5(0)$ & $72^{*}$ & $72^{*}$ \\
\hline 9 & $250(0)$ & $47,5(0)$ & $77^{*}$ & $78^{*}$ \\
\hline
\end{tabular}

\%SST: percentual extraído de sólidos suspensos totais; *Outliers.

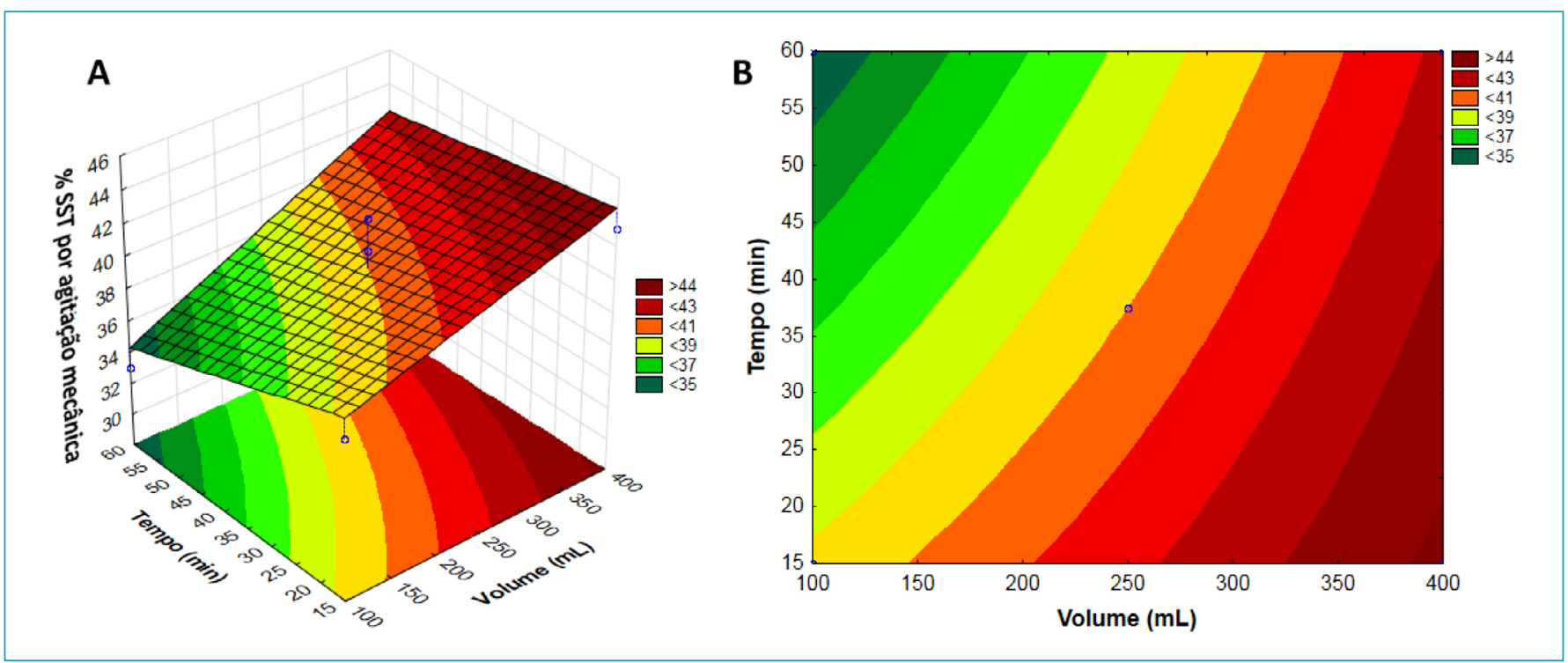

Figura 5 - Superfícies de resposta das variáveis volume $(\mathrm{mL})$, tempo (min) e percentual extraído de sólidos suspensos totais (\%SST) (Etapa I, Bloco 2 - Agitação mecânica). Variável resposta: \%SST.

superior (7\%) à extração com volume maior, sendo o mesmo resultado observado quando o tempo foi fixado em 90 min (Tabela 4).

Essa tendência de maior eficiência de extração em volumes menores quando o tempo é mantido constante (ainda que sem significância estatística) poderia ser justificada pelo efeito de a agitação no frasco de igual volume, mas com menor volume de água $(100 \mathrm{~mL})$, permitir maior movimentação e atrito entre as mídias, facilitando, assim, o desprendimento do biofilme, se comparado ao frasco com maior volume de água $(400 \mathrm{~mL})$.

Uma vez que a reprodução de um determinado resultado seja alcançada com precisão aceitável (ou seja, após validação), o cálculo do teor de biomassa em um MBBR poderá ser feito com base no percentual de biomassa removida das mídias via ultrassom, utilizando-se tal percentual para calcular o total de biomassa aderida por unidade de mídia e, em seguida, multiplicando-se pelo número total de mídias no reator. No presente estudo, $81 \pm 1$ mídias $/ \mathrm{L} \times 120 \mathrm{~L}=9.720$ mídias. Essa é a forma para estimar a biomassa que deverá ser validada em experimentos futuros.

Alguns estudos de operação e avaliação de MBBRs fazem menção à dificuldade em se alcançar uma remoção satisfatória da biomassa aderida às mídias, mesmo com o uso de substâncias químicas (ZHANG et al., 2013a; ZHANG et al., 2013b). Outros trabalhos relatam o uso 
de $\mathrm{NaOH}$ a 2M (OOI et al., 2018) ou a 3M (ABTAHI et al., 2018), sem registro sobre a eficácia do método. Alguns trabalhos fazem uso de ácido sulfúrico $\mathrm{H}_{2} \mathrm{SO}_{4} 5 \mathrm{~N}$ (FÅLAS et al., 2013) e ácido crômico $\mathrm{Cr}-\mathrm{H}_{2} \mathrm{SO}_{4}$ (LEVSTEK; PLAZL, 2009) e tampouco comentam o motivo para a escolha de tal método. As técnicas mais brandas com base na extração mecânica e no ultrassom também não avaliam a eficácia na remoção (DI BIASE et al., 2018; WANG; WEN; QIAN, 2005). Com base na literatura, portanto, é difícil traçar comparações acerca da eficiência dos diferentes métodos. A aplicação de otimização em processos de extração de biomassa aderida às mídias e o estudo comparativo deles são, portanto, totalmente justificados, evidentemente com preferência por métodos que não geram efluentes perigosos.

\section{CONCLUSÕES}

Os resultados obtidos com os desenhos experimentais aplicados e com os níveis das variáveis estudadas indicaram que o método de extração por ultrassom foi mais eficiente do que o método de extração por agitação mecânica na remoção da biomassa aderida às mídias. Além disso, o T de exposição ao ultrassom foi a variável que contribuiu positiva e significativamente para a eficiência de extração da biomassa expressa em \%SST (melhor resultado obtido no tempo máximo de $90 \mathrm{~min}$ ). Como o volume de água nos frascos não contribuiu significativamente para a extração do biofilme, recomenda-se a utilização do volume de mais fácil operacionalização dos proces$\operatorname{sos}(250 \mathrm{~mL})$ em razão da facilidade de manuseio e filtração. Alguns ensaios adicionais são recomendados, tendo em vista a padronização de um método para quantificação do biofilme sem utilização de ácidos fortes e geração de resíduos perigosos. Quanto à quantificação de SST utilizando-se diferentes membranas - tendo em vista que não há diferença significativa entre membranas de porosidade $0,7 \mathrm{e}$ $1,5 \mu \mathrm{m}$-, em razão da maior facilidade de filtração, recomenda-se o uso da membrana de maior porosidade.

\section{AGRADECIMENTOS}

Os autores agradecem à Coordenação de Aperfeiçoamento de Pessoal de Nível Superior - CAPES (processo n 1533268) a bolsa de doutorado concedida ao primeiro autor; à Fundação Carlos Chagas Filho de Amparo à Pesquisa do Estado do Rio de Janeiro - FAPERJ (processo $\mathrm{n}^{\circ}$ E-26/202.894/2018) e ao Conselho Nacional de Desenvolvimento Científico e Tecnológico - CNPq (processo n 435883/2018-6; processo $\mathrm{n}^{\circ} 308335 / 2017-1$ ) os recursos concedidos a projetos coordenados pela última autora; e à Financiadora de Estudos e Projetos - FINEP (CT-Infra Proinfra 01/11 Convênio 01.14.0081.03) os recursos concedidos à Universidade do Estado do Rio de Janeiro (UERJ). Agradecem também à Companhia Estadual de Águas e Esgotos do Rio de Janeiro (CEDAE) a parceria por meio de Convênio UERJ-CEDAE.

\section{REFERÊNCIAS}

ABTAHI, S.M.; PETERMANN, M.; FLAMBARD, A.J.; BEAUFORT, S.; TERRISSE, F.; TROTOUIN, T.; CASSAN, C.J.; ALBASI, C. (2O18) Micropollutants removal in tertiary moving bed biofilm reactors (MBBRs): Contribution of the biofilm and suspended biomass. Science of the Total Environment, v. 643, p. 1464-1480. https://doi. org/10.1016/j.scitotenv.2018.06.303

ANDREOTTOLA, G.; FOLADORI, P.; RAGAZZI, M.; TATÀNO, F. (2OOO) Experimental comparison between MBBR and activated sludge system for the treatment of municipal wastewater. Water Science \& Technology, v. 41, n. 4-5, p. 375-382. https://doi.org/10.2166/ wst.2000.0469

AMERICAN PUBLIC HEALTH ASSOCIATION (APHA); AMERICAN WATER WORKS ASSOCIATION (AWWA); WATER ENVIRONMENT FEDERATION (WEF). (2017) Standard methods for the examination of water and wastewater. 23a ed. Washington, D.C.: APHA. 1504 p.

BARWAL, A.; CHAUDHARY, R. (2014) To study the performance of biocarriers in moving bed biofilm reactor (MBBR) technology and kinetics of biofilm for retrofitting the existing aerobic treatment systems: A review. Reviews in Environmental Science and Biotechnology, v. 13, n. 3, p. 285-299. https://doi.org/10.1007/s11157014-9333-7

BASSIN, J.P.; DIAS, I.N.; CAO, S.M.S.; SENRA, E.; LARANJEIRA, Y.; DEZOTTI, M. (2016) Effect of increasing organic loading rates on the performance of moving-bed biofilm reactors filled with different support media: Assessing the activity of suspended and attached biomass fractions. Process Safety and Environmental Protection, v. 100, p. 131-141. https://doi.org/10.1016/j.psep.2016.01.007

CAO, S.M.S. (2014) Remoção biológica de matéria orgânica e nitrogênio de um efluente industrial em sistema de Leito Móvel com Biofilme de dois estágios. Dissertação (Mestrado) - Universidade Federal do Rio de Janeiro, Rio de Janeiro.

DI BIASE, A.; DEVLIN, T.R.; KOWALSKI, M.S.; OLESZKIEWICZ, J.A. (2018) Performance and design considerations for an anaerobic moving bed biofilm reactor treating brewery wastewater: impact of surface area loading rate and temperature. Journal of Environmental Management, v. 216, p. 392-398. https://doi. org/10.1016/j.jenvman.2017.05.093 
FALÅS, P.; LONGRÉE, P.; JANSEN, J.C.; SIEGRIST, H.; HOLLENDER, J.; JOSS, A. (2013) Micropollutant removal by attached and suspended growth in a hybrid biofilm-activated sludge process. Water Research, v. 47, n. 13, p. 4498-4506. https://doi.org/10.1016/j. watres.2013.05.010

FERRAI, M.; GUGLIELMI, G.; ANDREOTTOLA, G. (2010) Modelling respirometric tests for the assessment of kinetic and stoichiometric parameters on MBBR biofilm for municipal wastewater treatment. Environmental Modelling and Software, v. 25, n. 5, p. 626-632. https://doi.org/10.1016/j.envsoft.2009.05.005

JORDÃO, E.P.; PESSÔA, C.A. (2014) Tratamento de Esgotos Domésticos. 7 a ed. Rio de Janeiro: ABES.

LEVSTEK, M.; PLAZL, I. (2009) Influence of carrier type on nitrification in the moving-bed biofilm process. Water Science and Technology, v. 59, n. 5, p. 875-882. https://doi.org/10.2166/ wst.2009.037

MAHENDRAN, B.; LISHMAN, L.; LISS, S.N. (2012) Structural, physicochemical and microbial properties of flocs and biofilms in integrated fixed-film activated sludge (IFFAS) systems. Water Research, v. 46, n. 16, p. 5085-5101. https://doi.org/10.1016/j. watres.2012.05.058

NOGUEIRA, B.L. (2013) Modelagem matemática de reatores de leito móvel com biofilme para tratamento de efluentes. Dissertação (Mestrado) - Universidade Federal do Rio de Janeiro, Rio de Janeiro.

ØDEGAARD, H. (2006) Innovations in wastewater treatment: the moving bed biofilm process. Water Science \& Technology, v. 53, n. 9, p. 17-33. https://doi.org/10.2166/wst.2006.284

OLIVEIRA, D.V.M. (2008) Caracterização dos Parâmetros de Controle e Avaliação de Desempenho de um Reator Biológico com Leito Móvel (MBBR). Dissertação (Mestrado) - Universidade Federal do Rio de Janeiro, Rio de Janeiro.

OOI, G.; TANG, K.; CHHETRI, R.K.; KAARSHOLM, K.M.S.; SUNDMARK, K.; KRAGELUND, C.; LITTY, K.; CHRISTENSEN, A.; LINDHOLST, S.; SUND, C.; CHRISTENSSON, M.; BESTER, K.; ANDERSEN, H.R. (2O18)
Biological removal of pharmaceuticals from hospital wastewater in a pilot-scale staged moving bed biofilm reactor (MBBR) utilizing nitrifying and denitrifying processes. Bioresource Technology, v. 267, p. 677-687. https://doi.org/10.1016/j.biortech.2018.07.077

RODRIGUES, M.I.; IEMMA, A.F. (2009) Planejamento de experimentos \& otimização de processos. $2^{\mathrm{a}}$ ed. Campinas: Casa do Espírito Amigo Fraternidade Fé \& Amor. 358 p.

RUSTEN, B.; EIKEBROKK, B.; ULGENES, Y.; LYGREN, E. (2006) Design and operations of the Kaldnes moving bed biofilm reactors. Aquacultural Engineering, v. 34, n. 3, p. 322-331. https://doi. org/10.1016/j.aquaeng.2005.04.002

SHORE, J.L.; M'COY, W.S.; GUNSCH, C.K.; DESHUSSES, M.A. (2012) Application of a moving bed biofilm reactor for tertiary ammonia treatment in high temperature industrial wastewater. Bioresource Technology, v. 112, p. 51-60. https://doi.org/10.1016/j. biortech.2012.02.045

WANG, R.-C; WEN, X.-H.; QIAN, Y. (2005) Influence of carrier concentration on the performance and microbial characteristics of a suspended carrier biofilm reactor. Process Biochemistry, v. 40, n. 9, p. 2992-3001. https://doi.org/10.1016/j.procbio.2005.02.024

WANG, X;; BI, X:; HEM, L.J.; RATNAWEERA, H. (2018) Microbial community composition of a multi-stage moving bed biofilm reactor and its interaction with kinetic model parameters estimation. Journal of Environmental Management, v. 218, p. 340-347. https://doi.org/10.1016/j.jenvman.2018.04.015

ZHANG,S.; WANG, Y.; HE,W.; WU, M.: XING,M.; YANG, J.;GAO,N.:SHENG, G.; YIN, D.; LIU, S. (2013a) Linking nitrifying biofilm characteristics and nitrification performance in moving-bed biofilm reactors for polluted raw water pretreatment. Bioresource Technology, v. 146, p. 416-425. https://doi.org/10.1016/j.biortech.2013.07.056

ZHANG, S.; WANG, Y.; HE, W.; WU, M.; XING, M.; YANG, J.; GAO, N.: YIN, D. (2O13b) Responses of biofilm characteristics to variations in temperature and $\mathrm{NH} 4+-\mathrm{N}$ loading in a moving-bed biofilm reactor treating micro-polluted raw water. Bioresource Technology, v. 131, p. 365-373. https://doi.org/10.1016/j.biortech.2012.12.172 